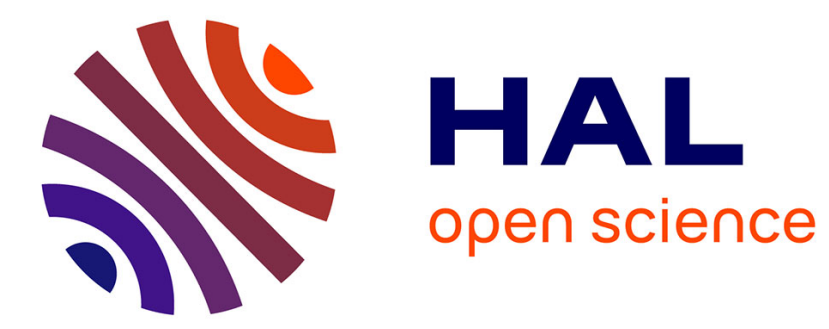

\title{
Inline measurement strategy for additive manufacturing
}

\author{
Matthias Bordron, Charyar Mehdi Souzani, Olivier Bruneau
}

\section{To cite this version:}

Matthias Bordron, Charyar Mehdi Souzani, Olivier Bruneau. Inline measurement strategy for additive manufacturing. Proceedings of the Institution of Mechanical Engineers, Part B: Journal of Engineering Manufacture, In press, 10.1177/0954405418755826 . hal-01710074

\section{HAL Id: hal-01710074 \\ https://hal.science/hal-01710074}

Submitted on 15 Feb 2018

HAL is a multi-disciplinary open access archive for the deposit and dissemination of scientific research documents, whether they are published or not. The documents may come from teaching and research institutions in France or abroad, or from public or private research centers.
L'archive ouverte pluridisciplinaire HAL, est destinée au dépôt et à la diffusion de documents scientifiques de niveau recherche, publiés ou non, émanant des établissements d'enseignement et de recherche français ou étrangers, des laboratoires publics ou privés. 


\title{
Inline Measurement Strategy for Additive Manufacturing
}

\author{
Matthias Bordron ${ }^{1}$, Charyar Mehdi-Souzani ${ }^{1}$, Olivier Bruneau ${ }^{1}$ \\ ${ }^{1}$ Lurpa, ENS Paris-Saclay, Univ. Paris-Sud, Univ. Paris 13, Sorbonne Paris Cité, Université Paris-Saclay, \\ 94235 Cachan
}

Corresponding author: charyar.souzani@ens-paris-saclay.fr

\begin{abstract}
Additive manufacturing (AM) takes a growing place in industry tanks to its ability to create free form parts with internal complex shape. Yet the final surfaces quality of the AM parts is still a challenge since it doesn't reach the required level for final use. To address this issue it is necessary to measure the form and dimensions deviation in order to plan post-process operations to be considerate. More over in a context of industry 4.0, this measurement step should be fully integrated into the manufacturing line as close as possible to the AM process and post-process. We introduce in this article an inline measurement solution based on a robot combined with a laser sensor. Robot allows reaching most of the orientation and positions necessary to digitize complex parts in a short time. The use of robot for digitizing is already addressed but not for metrological applications. Robots are perfectly design for velocity, ability and robustness but since now their poor positioning accuracy in not compatible with measuring requirements. The strategy adopted in this article is to provide an algorithm to generate path planning for digitizing AM parts at a given quality of the resulting cloud of points. After a discussion about the geometric and elastic model of the robot to identify the one that answers the quality requirements, the performances of the robot are evaluated. Thus several performances maps are introduced to characterize the behavior of the robot in its working volume. The qualification of the digitizing sensor is also performed to identify relation between digitizing parameters and the quality of final cloud of points. By using data resulting from the qualifications of sensor and robot and the part's CAD model the algorithm allows generating path planning to ensure the finale quality necessary to measure the shape deviation.
\end{abstract}

Key words: Digital manufacturing, Inline digitizing, Additive Manufacturing, optical measurement, robot, quality control, path planning, post-processing.

\section{Introduction}

Additive manufacturing (AM) is a rising field in industry because of its convenience to create complex parts. Many recent reports from governmental agencies, European Commission ${ }^{1}$ or national research institutions ${ }^{2,3}$ have shown the increasing place of AM technology and its economic impact in near future. It is now generally accepted that AM represents such a change that requires to rethink of the entire design, manufacturing and control chain ${ }^{4}$. Despite many advantages, today it is still not reasonable to consider a part obtained by $\mathrm{AM}$ as a finished ready-to-use part, this is particularly true 
for metal additive manufactured parts ${ }^{5}$. Additional manufacturing operations as post-processing with complementary process as machining are usually necessary to have a functional component. AM, can be defined as the process that allows building a 3D part by joining material layer by layer thank to a digital definition based on the CAD model ${ }^{6}$ and as opposed to subtractive manufacturing technologies. It is therefore by nature that this technology generates shape deviation on parts. Shape deviation and texture are the most noticeable undesirable external effects on additive manufactured parts. Those effects are generated by many different sources but the height of each layer and the relaxation of thermal stresses 7,8 play a major role. In this context it is necessary to have a metrological feedback of the parts for the quality control and the post-process planning. The measurement step can be considerate as the control step between two manufacturing processes (figure 1).

Figure 1. Measurement cell integrated on an AM production line

It allows characterizing the shape deviation of additive manufactured parts, information that will be used to select and defined the characteristics of the next manufacturing process necessary. Indeed it is often necessary to plan a post processing step with subtractive manufacturing techniques to polish, to reduce the surface stresses or just to reach the required tolerances that are not reachable with $\mathrm{AM}^{9}{ }^{9}$. In order to scale-up the production and to keep the cadence of production an in line measurement process would be the best option. Classical CMM technology provides probably the best metrological feedback but due to the time consuming it's not possible to use it for an inline measurement specially when it is added to an AM chain where the measurement of each part is necessary for planning of the post-processing. We propose here the use of a robot combined with a Laser Triangulation Sensor (LTS) to directly measure each part on the production line with few assembly/disassembly compared to an equivalent CMM measurement.

Robots have poor pose performances that limit their use for high precision task especially for measurement applications. But they offer a great flexibility of movement with six degrees of freedom 
(DoFs) and a high speed of execution. Those characteristics stay very attractive and could allow the 3D digitizing of complex parts made by additive manufacturing with the continuous reorientation provided by robots. Hence the robot facilitates the integration of the measurement process on production lines. Yet using a robot for such application is impossible until its pose accuracy is improved, as defined in ISO 9283 and confirmed by industrial needs. ${ }^{10,11}$

In this context this paper introduces the development of a 3D digitizing cell using a robot as displacement and a LTS for parts measurement. The specifications on the part to be produced by additive manufacturing induce digitizing quality requirements to carry out a geometrical measurement and to address the post-process planning. A strategy is addressed to use the robot for digitizing parts with the required quality of digitized points. This strategy also pays attention to the digitizing time to minimize it. This results in a digitized part with a given quality and an optimized speed. The final digitizing time and quality highly depends on the robot and on the LTS performances, so we study both in this paper. The calibration of laser sensors is well known ${ }^{12}$, however there is no standard to measure LTS performances and we must adapt methods. ${ }^{13}$ Robot performances are deduced from experiments and models, but models used for industrial robots are usually simple geometric models using the $\mathrm{DH}$ convention ${ }^{14,15}$ plus one stiff parameter for the angular elasticity. This is not enough for an accurate description of robot behavior so we investigated a detailed and original geometric and elastic model. We also experiment an original method for a quick and convenient identification of model parameters. Robot performances are heterogeneous in robot workspace ${ }^{16}$, so we have to choose a work zone to realize the digitizing where robot performances correspond to quality and speed needs. This can be done with a constrained path ${ }^{17}$, so a pathplanning algorithm is introduced, based on robot performance cartography and on LTS performances. The aim is to reach the quality targeted for additive manufacturing by using the robot and the LTS in their best configurations. The introduced strategy allows thus to create a path that guarantee the quality requirement on the points digitized and that optimize the digitizing time or other criteria like the energy consumption (not investigated here). 
An external measurement system is used to control the path followed by the robot so as to respect the quality of the resulting digitizing. It allows correcting the path and, used with the robot model, it brings a redundancy that provides a more robust result. By this way the quality of robot positioning is not an issue anymore since the positioning is provided by the external system and thus its quality is depending of the accuracy of the latter one.

After introducing the components of the digitizing system, the global strategy for path planning is presented. The third section presents the geometric and elastic model of the robot and in the fourth one we detail the performance indexes helpful for the path planning to exploit robot capabilities. The last section deals with the LTS calibration and integration as well as the use of the external measurement system before ending by a conclusion and some perspectives about future work.

\section{Path planning strategy for a robotized digitizing}

The robot six DoFs allow a complete reorientation of the LTS and thus a more flexible path to digitize the complex parts made by additive manufacturing. But we also need to respect digitizing requirements and to optimize other criteria like the digitizing time, the energy consumption or maybe the final digitizing quality. For those reasons, we detail here the path planning algorithm and the integration of robot performances and LTS capabilities in this algorithm. It results in the calculation of the best path depending on robot and sensor performances.

\subsection{Optical measurement and robotized digitizing cell for additive manufacturing}

With additive manufacturing many new challenges have emerged from a metrological point of view. In stet: The assessment of geometrical capacity of AM system by using metrological artifact ${ }^{18,19}$; In process measurement for monitoring the laying of materials during the manufacturing and off-course the shape deviation measurement for quality control and post-processing planning ${ }^{9}$. In this paper we will focus on the last point for quality control. There are many different technologies for optical 
forms measurement, but laser triangulation and structured light projection are the most appropriated and offer the best compromises between volume measurement, time consuming and accuracy required by industries such as aerospace or aerospace ${ }^{13,20}$. In this paper the part digitizing will be performed thanks to a laser sensor. This solution is more flexible than structured light projection for the measurement of complex and freeform surfaces, which characterized the parts generated by AM. Indeed the laser plane can be easily oriented and thus facilitates the accessibility of the whole part. This is especially true if a robot is used as a displacement system. Many applications integrate robots to use some of their advantages, for example a lot has been done in machining with robots. ${ }^{21,22,23}$. Brosed ${ }^{24}$ uses a robot for a digitizing but only exploits it to carry, position and orient parts. The robot behavior (model and performances) is not taken into account in the path planning process, and many robot capabilities are unused.

The path planning is essential to improve the robot posing as it allows taking advantage of its six

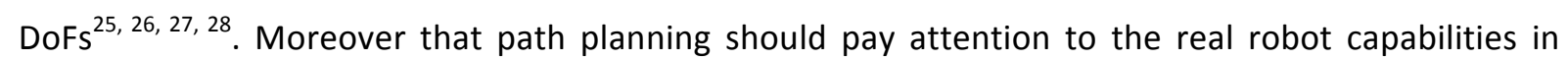
terms of speed or posing quality, so it should be based on robot performance indexes ${ }^{16}$. But as related by $\mathrm{Zha}^{28}$, most of path generators do not take into account performance indexes. The robot's model used for the path planning also plays a significant role in the resulting quality of the digitized points. And performance indexes are mostly calculated with the robot model. This is the reason why the selection and the definition of an adapted robot model are a major concern ${ }^{29,30}$ developed in this paper.

In this context we introduce a digitizing cell (Figure 2), which uses a robot and two contactless sensors: one for the part digitizing and one for an external control of the robot path.

Figure 2. Multi-sensors digitizing cell

This cell involves the use of a global strategy resulting in digitized points respecting the additive manufacturing needs while taking advantage of the robot's capabilities. This strategy is developed in two parts: the path planning related to robot and LTS performances, and the deviation control of the 
robot end-effector. Considering the small field of view of the CTrack, we allow it to lose the track of the end-effector. The path is corrected later when the effector comes back in the CTrack vision field. In this case, the path is regularly corrected during the process. In order to increase the correction frequency (for instance, the sample time), we intend to equip the cell with more advanced tracking devices.

\subsection{Strategy for path planning algorithm}

The path planning algorithm gives the best trajectory regarding the quality requirements on the resulting cloud of points. The obtained path is actually a set of path points, each point is characterized by a position and an orientation of the robot end-effector. When the robot CNC reads the planned path, it uses the robot model to convert points from the Cartesian space (position and orientation) to the joint coordinate space (six angles for robot articulations). During the execution of the trajectory, more path points will be created from the existing points, by linear interpolation (to smooth the trajectory).

The Figure 3 describes the path planning process. It starts with the translation of the additive manufacturing needs in terms of requirements on the digitizing quality. A CAD part is also needed for the process as it provides the part geometry. Then, the sensor performances knowledge allows us to transform the digitizing requirements into a local path specification, giving the digitizing distance and the angle between the sensor and the part. Then we look for areas in the robot workspace where the path could take place with the local path specifications. So multiple areas are found where sets of path points are partly defined: the local specifications partly fixed the position and orientation of the path points. Among the admissible areas, and with the remaining freedom on path point positions and orientations, we look for the best path in terms of speed or robot posing quality. Finally we pick the best path among the optimized path from each area.

Figure 3. Path generation strategy for digitizing 
To apply this strategy, a perfect knowledge of the LTS characteristics is essential. The laser sensor used for digitizing is a KREON KZ25. We evaluated its performances thanks to an assessment protocol: QualiPSO ${ }^{13}$. The next section introduces a model of the robot used to calculate robot performances but also used to generate the final robot path in Figure 3.

\section{Geometric and elastic robot model}

The path planning strategy is used to optimize the speed of the digitizing process and to respect a high level of tolerancing requirements imposed by functional use of the additive manufacturing parts. But this strategy relies on the accuracy of the robot model used in the algorithm. Actually the robot model defines the relation between the Cartesian space and the joint space. The path is planned in the Cartesian space but the robot motion is defined in the robot joint space which explains the need for a model. So a robot model not detailed enough would take away the resulting path from the planned path and would lower the quality of the digitizing points. Furthermore many robot performances depend on the robot model as presented later on the fourth section. The digitized point's quality and the robot performance indexes calculation put this model as the main basis of our strategy. The model used by Kuka into the robot's computer numerical control (CNC) to generate path is poorly detailed in terms of robot defaults like deformations and backlashes. In order to represent as close as possible the real robot behavior, we propose a model that takes into account geometric defaults coming from manufacturing limits and assembly as well as non-geometric ones.

\subsection{Model selection and definition}

In literature, the Denavit-Hartenberg (DH) model is the best-known geometric parameterization convention ${ }^{14}$. It is mostly used with a different convention: the DH modified model, as it is more convenient ${ }^{31}$. It is often completed with the Hayati parameter, which handles consecutive parallel $a_{x e s}^{32}$. As geometric models are well known, we focus more on elastic models representing nongeometric defaults. Many elastic models are based on the $\mathrm{DH}$ parameterization, completed with 
some non-geometric defaults ${ }^{33,34,35}$. To define an accurate and complete model, three techniques go further:

- The FEA model (Finite Elements Analysis) uses finite elements to compute robot elastic deformations $s^{36,37}$.

- The MSA model (Matrix structural Analysis) uses the Euler-Bernoulli beam theory to compute the deformations of the robot links ${ }^{38}$.

- The VJM model (Virtual Joint Model) uses virtual springs with six DoFs where all displacements and backlashes are concentrated ${ }^{23}$.

We need a flexible model, able to take into account all significant robot defaults on the digitizing quality, so we choose an adaptable model that can contain any default: the VJM model. It is initially only made for deformations in articulations. Thus we modified the six DoFs virtual springs into a translation vector and a rotation vector, for both deformations and backlashes and applied to both links and articulations. The resulting model is adaptable and can be completed with more detailed models of joints and links 39,40 .

The VJM model is based on the DH geometric parameterization convention, and adds its virtual joints between the geometric components of the model (links and articulations) as explained in Figure 4. Our modified VJM model can handle all non-geometric phenomena into the translation and rotation vectors of its virtual joints. So now we need to model deformations and backlashes of robot joints and links to put them into virtual joints.

Figure 4. Calibration strategy for an elastic and geometric model of the robot, based on the modified VJM adapted model

The robot links are modeled using the beam theory from the MSA model ${ }^{38}$. For each link there are five stiff parameters and one parameter for the mass, which allow to express the link deformation under an external load. 
A similar theory does not exist for joint modeling as joints are commonly less detailed. So we use the standard ISO 230-1, in a polar frame to get four error terms ${ }^{41}$ :

- $d_{r}$ radial error motion

- $d_{z}$ axial error motion

- $d_{r}$ tilt error motion

- $d_{z}$ angular positioning error motion

Each of them includes a deformation and a backlash to get a total of eight parameters for each joint.

Yet we need to pay attention to special issues. Actually we cannot model all links with the beam theory. For those that does not respect beam conditions, we use a FEA model. Otherwise, since the 3rd link and the 4th link are necessarily aligned, their length cannot be identified separately.

\subsection{Model calibration: identification of parameters}

The external measurement system (Figure 2 ) is used to identify all model parameters that is to say geometric and elastic parameters. Identifying parameters with a contactless method is more convenient as digitizing can be done on production lines. Parameters must represent the robot behavior in its entire workspace and not only in few spots used for the identification by most calibration methods $^{12}$. For this reason, we develop here the circle point analysis (CPA) method ${ }^{42}$ that uses the entire workspace.

In this method, the positions of robot joints are identified separately. The joint moves in the field of view of the external measurement system that follow a target placed on the end-effector. Then the circle's arc measured allows finding the joint position. The CPA identifies all joints locations and we calculate the robot parameters between joints. This method gives good joints positions as we follow a joint in its complete availability range, so we get accurate parameters. Contrary to classic methods, we don't try to minimize of position errors in few points, but we directly estimate robot parameters. 
The CPA identification is initially made for geometric calibration, so we adapted this method for elastic parameters identification. To do so, the identification experiments were done while modifying the efforts on the robot end-effector.

The calibration of the robot parameters with the CPA method follows the strategy presented on the Figure 4. A first estimation of geometric parameters is done by assuming the robot components as rigid. Then we use it as an input for the first iteration to get elastic and geometric parameters. The calculation goes on until a given accuracy is reached by all parameters. The estimation of elastic parameters is done with the Levenberg Marquardt algorithm. Once parameters are identified, the robot model is exploited for path planning and for performance indexes calculation.

\section{Definitions and uses of robot performance indexes}

As mentioned in the second section, the result of the path planning algorithm will be a trajectory made of path points. Those path points give successive positions and orientations for the robot effector in the robot Cartesian work-space. Robot performances are needed to find possible path with the robot according to digitizing requirements and to LTS capabilities. This results in multiple possible paths respecting the quality needed for the digitizing of additive manufacturing parts. And for each path, the path points are not totally fixed: successive positions and orientations can change a bit while still respecting requirements. So robot performances also help to select among all possibilities the best path and the best path points (positions and orientations) in terms of digitizing speed or quality. For this optimization we use performance indexes mostly based on the robot model. So it is interesting to see how the quality of indexes calculation depends on the quality of the robot model introduced in the previous section.

The assessment of robot performances permits to generate cartographies that help us to respect digitizing quality and to optimize the digitizing time, so performances need to describe robot speed and positioning quality. In order to handle the random part of robot posing, we need a performance index on the robot fidelity to follow a path. So among existing indexes ${ }^{16}$, we look for quality and 
speed related indexes as well as other criteria indirectly linked to speed or quality. And during the path planning process, all indexes will be taken into account with different weights according to their importance for the calculation.

\subsection{A flexible path by minimizing articulation availability}

The articulation availability was firstly used by Liegeois to keep joints away from their limits, that is to say to keep an amount of available movement ${ }^{43}$. Trying to maintain this index high results in a smooth and flexible path. Yet this index does not handle the singular configurations, so it must be used carefully and with other indexes like manipulability or the condition number.

This index requires the knowledge of joints limits to compare them with the current joint position as on equation (4.1). Then the index is a mean of each joint position divided by their limits as shown on equation (4.2).

$$
\begin{aligned}
\mathrm{D} q_{i} & =\left|q_{i}-\frac{q_{i \max }+q_{i \min }}{2}\right| \\
D & =\frac{1}{n} \sum_{1}^{n}\left(\frac{\Delta q_{i}}{\Delta q_{i \max }}\right)^{2}
\end{aligned}
$$

As this index helps to stay away from joint limits, its cartography represents the robot reachability like on Figure 5 where the index can vary from 0 (best availability) to 1 . This allows quickly eliminating unreachable workspace areas and simplifying the path planning.

Figure 5. Articulation availability for a vertical orientation

\subsection{Manipulability index for robot speed and quality}

The manipulability index was created by Yoshikawa to represent robot speed or posing quality ${ }^{44}$. It calculates the robot speed capability for a given configuration and depending on each joint speed capability. This calculation of the manipulability index $W$ presented in equation (4.3), requires the 
singular values of $J$ the jacobian matrix of the robot complete model (geometric and elastic). There is one singular value $s_{i}$ per joint $i(i=1, . ., 6)$, so we see how each joint contributes to this index.

$$
w=|\operatorname{det}(J)|=s_{1} \cdot s_{2} \cdot s_{3} \cdot s_{4} \cdot s_{5} \cdot s_{6}
$$

There is another use than speed capability control for this index. A high manipulability means that little move of joints generate a big movement of the effector, and so, a very bad resolution. Conversely, a bad manipulability means a good resolution on the end-effector movement. Thus, the manipulability inverse represents the robot accuracy and the manipulability index can be simultaneously used to determine robot speed capability and robot posing quality.

Since the equation (4.3) uses the Jacobian matrix, the manipulability calculation requires the use of the robot model. In order to ensure the quality of the manipulability index we need a model close to the real robot behavior, which means a good modeling and a good calibration. We see again the importance of the robot model inside the path planning process. For a vertical orientation of the end-effector, the figure 6(a) illustrates a cartography of the manipulability index calculated with the identified robot model in an area limited by the articulation availability

\subsection{Condition number to control the path generation quality}

Khan and Angeles introduced the condition number to define the matrix conditioning and thus limit error propagation in calculation ${ }^{45}$. The condition number calculated in equation (4.4) is based on the jacobian matrix of the robot model, which makes another index dependent on the model.

$$
K(U)=\frac{1}{6} \sqrt{\left.\operatorname{tr}\left(U J^{T}\right) \cdot \operatorname{tr}\left(U \cdot J^{T}\right)^{-1}\right)}=\frac{S_{\max }}{S_{\min }}(4.4)
$$

This index is used for many purposes: accuracy, dexterity or error propagation. For instance it helps seeing the velocities disparities between joints. A low index means a good repartition of velocity capability among joints and a high index shows that an axis is near its speed limit. The figure $6(b)$ shows a cartography of this index for a vertical orientation of the end-effector in an area limited by 
the articulation availability. We can see that areas to avoid for good matrix conditioning are clearly situated.

Figure 6. (a) Robot manipulability cartography for a fixed orientation of the end-effector. This cartography shows areas where the robot can generate high speeds. (b) Condition number cartography through the robot working area

\subsection{End-effector repeatability with the robot repeatability model}

In robotic vocabulary, the repeatability is the capacity of a robot to leave and return exactly on a point, that is to say the uncertainty of robot posing. This capacity is measured on few points in the robot workspace and the mean value corresponds to the robot repeatability. This is a global value for the entire workspace so it does not represent the true robot behavior, especially since the repeatability capacity highly depends on the current robot configuration. Thus we developed a repeatability model giving the robot repeatability in a given configuration, which is more accurate than an average value for all configurations. The basis of this model was done by Brethe who built the robot repeatability from the six joint repeatability values ${ }^{46}$, which are the capability of joints to return in an angle. We went further by also making joint repeatability models, depending on the forces on joints and the joint acceleration and speed. Finally we get an accurate model to calculate robot repeatability for a given configuration, however the identification of this model requires the identification of all parameters linking the joint repeatability to the joint forces, to the accelerations and to the speeds. We conducted a series of experiments to determine the more influential parameters on each joint behavior, depending on forces, speeds or accelerations.

The cartography of repeatability shows the most reliable areas where the robot will closely follow the path planned.

\section{Qualification and integration of sensors used in the digitizing cell}

In order to increase the quality require for the additive manufacturing application, we use an external measurement system to control the execution by the robot of the planned path. Moreover 
we use this system to calibrate the robot model, so the quality of the identified model parameters depends on this external system. This section focuses on the qualification of this system and of the used LTS.

\subsection{External measure of the digitizing}

The digitizing cell components are: a six DoFs robot as a displacement system (support), a laser triangulation sensor to digitize parts, and a stereovision system, the CTrack, as the external measurement system. This last component is a major part of the cell as it helps for many purposes in the path planning.

Firstly, the external measurement system is used to follow the robot path during execution and to control deviation, even though it lengthens the cycle-time with additional calculations ${ }^{47}$. The robot model and its calibration are well fitted but a correction of the path ensures the quality of the digitized points. The deviation control of the executed path is identified with the external measurement system, then it computes the correction and finally it generates a new path and sends it to the robot CNC. The total computation time must be short enough compared to the robot cycletime in order to really correct the path.

The robot joint coordinates (angles given by sensors) and the robot model allow computing the current configuration of the robot, which is given to the LTS to acquire digitized points. The CTrack can be used to replace the robot joint coordinate if sensors information is not accessible, or it can be used as a redundant way to reinforce sensors data.

Finally this external measurement system is used during the robot model calibration with the CPA method (described in the third section). So a good qualification of the CTrack is essential to be sure to obtain good robot parameters, and for all other uses of this system. Thus we check the adequacy of the external measurement system capabilities with requirements on the CPA method and on path controlling. For this purpose a specific assessment protocol has been carried out.

\subsection{Integration of the laser sensor}


The LTS has its own model and its own parameters that need to be identified for the configuration used thank to a calibration process. The calibration is an important step since it affects the quality of the final digitizing points. The sensor calibration can be divided in two parts: the internal and external calibration. The intrinsic parameters describe the geometry of the laser sensor components, they place the 2D CCD matrix (the camera) with regard to the laser plane and detail the camera characteristics (focal, resolution ...). This allows calculating the 3D coordinates of the point digitized in the sensor frame. The intrinsic parameters describe the sensor itself, they are commonly given by the constructor and the internal calibration process is not accessible to the user.

The LTS has extrinsic parameters that are used to place the sensor relatively to the displacement system (the robot). In order to integrate the LTS to the digitizing cell we need to find those parameters. Once we know the configuration of the LTS in relation to the robot, and with the knowledge of robot current configuration, it is possible to convert the 3D digitized point from the sensor frame to the global cell frame. To get extrinsic parameters, a calibration gage is used ${ }^{12}$.

The LTS integration can be done in two different ways: the sensor can be fixed to the robot support ${ }^{12}$, or the LTS can be static in the robot workspace and the robot can hold the part to digitize ${ }^{24}$. In most cases the sensor is carry by the robot but for inline measurement into a AM chain this solution is not optimum for two reasons: the measurement can not be performed with the part placed into the AM system due to accessibility issues and the necessity to remove the part from the AM system to the post-processing system. For these reasons we have introduced a digitizing cell where the part is removed from the AM system by the robot which run the digitizing path in front of the TLS before placing the part in the next post-process system.

Finally, we need to qualify the digitizing capacity of our digitizing cell. A metal benchmark part was designed with a specific geometry to test the cell capacity to digitize plans, angles, and curves. Then both LTS integration cases (static or mobile) are set up and the assessment start to identify the behaviors for: 
- The digitizing quality through the computation of the noise and the completeness on the generated cloud of points.

- The digitizing speed with or without our path optimization

- The flexibility of the cell to digitize complex parts

This specific qualification is made for this cell as its size prevents from using a classic method ${ }^{48}$. It helps us to know if the cell can digitize a given part made by additive manufacturing, regarding additive manufacturing specifications. Yet before those qualification experiments, a validation protocol must be carried out to ensure the validity of the path planning strategy and of the components calibration. We use a calibrated artifact and the external measurement system to compare the posing quality of the robot with our model and with the model used by Kuka in the robot's CNC. We begin with the same starting point and we calculate end points with both models at a theoretical distance equal to the artifact length. This is done for various configurations. Similar experiments are done to compare a classic path and a path optimized by our algorithm, by looking at the exact position of path points.

\section{Conclusion}

Additive manufacturing technology has open up new design possibility especially for free-form and complex parts. But up today the low surface quality and the form deviation that characterized parts generated by $\mathrm{AM}$ (especially for metal $\mathrm{AM}$ ) is a limitation to produce ready to use parts. In this context the control quality by measurement and for post-process planning is required. This paper is a first step to introduce a solution to integrate this measurement step into the manufacturing line which will avoid the part's disassembly and its transportation to and from the out-line classical measurement cell. It's reasonable to considerate that this solution will save time and will facilitate the post-treatment of AM parts. Moreover, thanks to the robot accessibility, the presented solution allows digitizing complex parts with none or few parts repositioning which also contribute to saves time. The path is generated automatically from the part CAD model and has an optimized speed. The 
capability to digitize with a robot for parts control in an additive manufacturing context is a request that would allow saving time and resources. Actually the measurement step needs to be flexible and convenient to be on the production line and to be able to digitize the produced complex parts. The most appropriate displacement solution is a six-axis robot that responds to those needs. Yet digitizing with robots is still few developed nowadays, and robots advantages are rarely exploited. Contrary to previous work, the strategy developed in this paper tends to take advantage of full robot performances and digitizing sensor capabilities to obtain optimized digitized points for a control application. To do so an optimized path is identified among an admissible set of paths previously calculated with robot and sensor capabilities. The path planning process focuses on the final required quality for digitized points and the digitizing time. In order to reach the level of quality compatible with the additive manufacturing specifications, geometric and elastic models have been defined to detail robot behavior. The digitizing cell is completed by an external measurement system used for path deviation control and for digitized point calculation in order to avoid the poor positioning of the robot. This strategy can also be applied to other applications needing a convenient way to control parts on a production line. For instance large parts like aeronautic parts currently require complex measurement devices ${ }^{49}$ with significant logistics, where our strategy could be applied.

\section{References}

1. Key Enabling Technologies D2 "Advanced Manufacturing Systems and Biotechnologies". Additive Manufacturing in FP7 and Horizon 2020, Report from the EC Workshop on Additive Manufacturing, 18 June 2014.

2. US Department of Commerce, NIST, Measurement Science Roadmap for Metal-Based Additive Manufacturing, May 2013.

3. Woolliams P, Halstead J, Morris A and Leach R, NPL, Additive Manufacturing Strategy, UK, 2014. 
4. Thompson M, Moroni G, Vaneker T, Fadel G, Campbell R, Gibson I, Bernard A, Schulz J, Graf P, Ahuja B, Martina F. Design for Additive Manufacturing: Trends, Opportunities, Considerations, and Constraints. CIRP Annals - Manufacturing Technology vol.65, iss.2, pp737-760, 2016.

5. Aidibe A, Tahan A and Brailovski V. Metrological Investigation of a Selective Laser Melting Additive Manufacturing System: A Case Study. IFAC-paper online 49-31 (2016) 25-29.

6. STM Standard, Standard terminology for additive manufacturing technologies, ASTM Standard F2792-12a. ASTM International, West Conshohocken, PA, 2012.

7. Moylan S, Slotwinski J, Cooke A, Jurrens K and Donmez MA. Lessons Learned in Establishing the NIST Metal Additive Manufacturing. NIST, 2013.

8. Roberts IA, Investigation of residual stresses in the laser melting of metal powders in additive layer manufacturing. Ph.D. thesis, University of Wolverhampton, 2012.

9. Stavroulakis PI and Leach RK. Invited Review Article: Review of post-process optical form metrology for industrial-grade metal additive manufactured parts. Review of Scientific Instruments 87, 041101 (2016).

10. Sansoni G, Trebeschi M and Docchio F. State-of-the-art and applications of 3D imaging sensors in industry, cultural heritage, medicine, and criminal investigation. Sensors 2009; 9(1): 568-601.

11. NF ISO 9283 Manipulating industrial robots - Performance criteria and related test methods.

12. Santolaria J, Guillomía D, Cajal C, Albajez JA and Aguilar JJ. Modelling and calibration technique of laser triangulation sensors for integration in robot arms and articulated arm coordinate measuring machines. Sensors 2009; 9(9): 7374-7396.

13. Mehdi-Souzani C, Quinsat $Y$, Lartigue $C$ and Bourdet P. A knowledge database of qualified digitizing systems for the selection of the best system according to the application. CIRP Journal of Manufacturing Science and Technology 2016; 13: 15-23. 
14. Denavit J. A kinematic notation for lower-pair mechanisms based on matrices. Trans. of the ASME, Journal of Applied Mechanics 1955; 22: 215-221.

15. Olabi A, Damak M, Bearee R, Gibaru O and Leleu S. Improving the accuracy of industrial robots by offline compensation of joints errors. Industrial Technology (ICIT), IEEE International Conference, March 2012, 492-497.

16. Moreno HA, Saltaren R, Carrera I, Puglisi L and Aracil R. Performance Indices for Robotic Manipulators: a review of the State of the Art. Revista Iberoamericana de Automática $e$ Informática Industrial RIAI 2012; 9(2): 111-122.

17. Vosniakos GC and Chronopoulos A. Industrial robot path planning in a constraint-based computer-aided design and kinematic analysis environment. Proceedings of the Institution of Mechanical Engineers, Part B: Journal of Engineering Manufacture, 2009, 223(5), 523-533.

18. Mehdi-Souzani C, Piratelli-Filho A, Anwer N. Comparative Study for the Metrological Characterization of Additive Manufacturing artefacts, JCM Conference, International Joint Conference on Mechanics, Design Engineering \& Advanced Manufacturing, Catania, ITALY, 14-16 sep. 2016.

19. Moylan S, Slotwinski J, Cooke A, Jurrens K, Donmez MA. Proposal for a standardized test artifact for additive manufacturing machines and processes. In: Proceedings of the 2012 annual international solid freeform fabrication symposium. 2012. p. 6-8.

20. Zuquete-Guarato A, Mehdi-Souzani C, Quinsat $Y$, Lartigue C and Sabri L. Towards a new concept of in-line crankshaft balancing by contact less measurement: process for selecting the best digitizing system. ASME 2012 11th Biennial Conference on Engineering Systems Design and Analysis, PP 17-25, Nantes, France, 2012.

21. Abele $E$, Weigold $M$ and Rothenbücher S. Modeling and identification of an industrial robot for machining applications. CIRP Annals-Manufacturing Technology 2007; 56(1): 387-390. 
22. Guillo M and Dubourg L. Impact \& improvement of tool deviation in friction stir welding: Weld quality \& real-time compensation on an industrial robot. Robotics and ComputerIntegrated Manufacturing 2016; 39: 22-31.

23. Pashkevich A, Klimchik A and Chablat D. Enhanced stiffness modeling of manipulators with passive joints. Mechanism and machine theory 2011; 46(5): 662-679.

24. Brosed FJ, Aguilar JJ, Guillomía D and Santolaria J. 3D geometrical inspection of complex geometry parts using a novel laser triangulation sensor and a robot. Sensors 2010; 11(1), 2010, 90-110.

25. Kim T and Sarma SE. Toolpath generation along directions of maximum kinematic performance; a first cut at machine-optimal paths. Computer-Aided Design 2002; 34(6): 453468.

26. Larsson S and Kjellander JAP. Motion control and data capturing for laser scanning with an industrial robot. Robotics and Autonomous Systems 2006; 54(6): 453-460.

27. Mineo C, Pierce SG, Nicholson PI and Cooper I. Robotic path planning for non-destructive testing-A custom MATLAB toolbox approach. Robotics and Computer-Integrated Manufacturing 2016; 37: 1-12.

28. Zha XF. Optimal pose trajectory planning for robot manipulators. Mechanism and Machine Theory 2002; 37(10): 1063-1086.

29. Elatta AY, Gen LP, Zhi FL, Daoyuan Y and Fei L. An overview of robot calibration. Information Technology Journal 2004; 3(1): 74-78.

30. Hollerbach JM. A survey of kinematic calibration. The robotics review 1 MIT Press, January $1989,207-242$.

31. Khalil W and Kleinfinger J. A new geometric notation for open and closed-loop robots. Robotics and Automation Proceedings, IEEE International Conference, April 1986, Vol. 3, 1174-1179. 
32. Hayati SA. Robot arm geometric link parameter estimation. Decision and Control, The 22nd IEEE Conference, December 1983, 1477-1483.

33. Driels MR and Pathre US. Generalized joint model for robot manipulator kinematic calibration and compensation. Journal of robotic systems 1987; 4(1): 77-114.

34. Sheth PN and Uicker JJ. A generalized symbolic notation for mechanisms. Journal of Engineering for Industry 1971; 93(1): 102-112.

35. Whitney DE, Lozinski CA and Rourke JM. Industrial robot calibration method and results. Department of Mechanical Engineering, MIT 1985.

36. Bouzgarrou BC, Fauroux JC, Gogu G and Heerah Y. Rigidity analysis of T3R1 parallel robot with uncoupled kinematics. Proc. of the 35th International Symposium on Robotics (ISR), Paris, France, March 2004.

37. Wang G, Dong $\mathrm{H}$, Guo $\mathrm{Y}$ and Ke $\mathrm{Y}$. Finite element simulation and experimental analysis of robotic boring based on an approach of equivalent stiffness. Proceedings of the Institution of Mechanical Engineers, Part B: Journal of Engineering Manufacture, 2017, 0954405416683430.

38. Khalil W and Besnard S. Geometric calibration of robots with flexible joints and links. Journal of Intelligent and Robotic systems 2002; 34(4): 357-379.

39. Li YT and Fan KC. A novel method of angular positioning error analysis of rotary stages based on the Abbe principle. Proceedings of the Institution of Mechanical Engineers, Part B: Journal of Engineering Manufacture, 2017, 0954405416688936.

40. Cheng $Q$, Sun B, Liu Z, Feng $Q$ and Gu P. Geometric error compensation method based on Floyd algorithm and product of exponential screw theory. Proceedings of the Institution of Mechanical Engineers, Part B: Journal of Engineering Manufacture, 2016, 0954405416663537.

41. NF ISO 230-1 Test code for machine tools - Part 1 : geometric accuracy of machines operating under no-load or quasi-static conditions. 
42. Santolaria J, Conte J and Ginés M. Laser tracker-based kinematic parameter calibration of industrial robots by improved CPA method and active retroreflector. IJAMT 2013; 66(9-12): 2087-2106.

43. Liegeois A. Automatic supervisory control of the configuration and behavior of multibody mechanisms. IEEE transactions on systems, man, and cybernetics $1977 ; 7(12): 868-871$.

44. Yoshikawa T. Manipulability of robotic mechanisms. The international journal of Robotics Research 1985; 4(2) 3-9.

45. Khan WA and Angeles J. The kinetostatic optimization of robotic manipulators: the inverse and the direct problems. Journal of mechanical design 2006; 128(1): 168-178.

46. Brethé JF and Dakyo B. A stochastic ellipsoid approach to repeatability modelisation of industrial manipulator robots. Intelligent Robots and Systems IEEE/RSJ International Conference on 2002; Vol. 2: 1608-1613.

47. Corke PI. High-performance visual closed-loop robot control. Doctoral dissertation, University of Melbourne, 1994.

48. Muelaner JE, Cai B and Maropoulos PG. Large-volume metrology instrument selection and measurability analysis. Proceedings of the Institution of Mechanical Engineers, Part B: Journal of Engineering Manufacture, 2012, 224(6), 853-868.

49. Peggs GN, Maropoulos PG, Hughes EB, Forbes AB, Robson S, Ziebart M and Muralikrishnan B. Recent developments in large-scale dimensional metrology. Proceedings of the Institution of Mechanical Engineers, Part B: Journal of Engineering Manufacture, 2009, 223(6), 571-595. 